\title{
Sodium butyrate induces senescence and inhibits the invasiveness of glioblastoma cells
}

\author{
HIDEMITSU NAKAGAWA ${ }^{1}$, SATORU SASAGAWA ${ }^{2}$ and KAZUYUKI ITOH ${ }^{2}$ \\ ${ }^{1}$ Department of Neurosurgery, Nozaki Tokushukai Hospital; ${ }^{2}$ Department of Molecular Biology, \\ Research Institute Nozaki Tokushukai, Daito, Osaka 574-0074, Japan
}

Received July 19, 2017; Accepted October 20, 2017

DOI: $10.3892 / \mathrm{ol} .2017 .7518$

\begin{abstract}
Sodium butyrate (SB), a short chain (C-4) saturated fatty acid, is present in the human bowel at increased concentrations $(\sim 2 \mathrm{mM})$ as a food metabolite. It has been demonstrated that SB exerts an anti-tumor effect as a histone deacetylase inhibitor; however, its precise mechanism of action remains to be elucidated. The present study focused on the mechanisms underlying the effects of SB on glioblastoma (GB) cell proliferation, motility and invasion. In human GB A172 cells, flow cytometry and a Boyden chamber assay demonstrated that physiological concentrations of SB (0.25-4.00 mM) dose-dependently inhibited cell proliferation and invasion. SB also affected cellular morphology, with increases in cell area and the number of focal adhesions observed. However, the phosphorylation (Y397 site) of focal adhesion kinase (FAK) was increased, while that of myosin light chain (S19 site) was unaltered. All of these SB-induced effects were reversible and attenuated following SB withdrawal. In addition, A172 cells treated with SB exhibited positivity for senescence-associated (SA) $\beta$-galactosidase (gal) staining and elevated protein expression of p53 and p21 in a time- and dose-dependent manner, whereas the expression of p21 mRNA decreased. Knockdown of $\mathrm{p} 21$ expression using small interfering RNA reversed the inhibition of cell growth inhibition and positivity for SA $\beta$-gal staining, but did not reverse the inhibition of cell motility and enhanced phosphorylation of FAK. This suggests that cells require p21 to induce senescence but not for SB-mediated decreased motility. Therefore, the current study demonstrated that SB inhibits GB cell proliferation, induces cells to senesce and inhibits tumor cell invasion, indicating that it may be developed as a novel therapeutic strategy to treat GB.
\end{abstract}

Correspondence to: Dr Kazuyuki Itoh, Department of Molecular Biology, Research Institute Nozaki Tokushukai, 2-10-50 Tanigawa, Daito, Osaka 574-0074, Japan

E-mail:k.itoh@tokushukai.jp

Key words: sodium butyrate, glioblastoma, senescence, invasion, targeted therapy

\section{Introduction}

The prognosis of patients with glioblastoma (GB) remains poor despite the use of multidisciplinary treatments, which consist of maximal surgical resection, radiotherapy and concomitant/adjuvant chemotherapy with temozolomide (1). The median time-to-recurrence following standard therapy is $\sim 7$ months (2). Once tumor recurrence has occurred (generally within 1 year), further clinical deterioration may occur, along with expansive tumor growth, as identified by contrast-enhanced imaging (3) and the dissemination of the tumor into the cerebrospinal fluid (4). Patients with GB typically succumb within 2 years of diagnosis, despite multiple types of therapy available to treat GB (5). Therefore, in order to combat this devastating disease, novel targeted therapies to treat patients with GB are strongly required. In addition, since GB appeared in the brain, therapies are required to minimize adverse effects on the neuronal cells to keep host brain function.

Sodium butyrate (SB), a short chain (C-4) saturated fatty acid, is present in the human bowel at increased concentrations ( $\sim 2 \mathrm{mM})$ as a food metabolite (6). It has been reported that SB exerts an anti-tumor effect as a histone deacetylase (HDAC) inhibitor in colon (6,7), pancreas (8) and liver cancer (9) as well as in glioma (10); however, its precise mechanism of action remains to be fully elucidated (10-12). The present study focused on the underlying mechanism of SB regarding its effects on human GB cell proliferation, motility and invasion.

In the present study, SB induced dose-dependent growth inhibitory effects in vitro with G1/S phase arrest and stabilization of $\mathrm{p} 21$ expression. SB also increased senescence-associated (SA) $\beta$-galactosidase (gal) levels and exhibited a significant inhibitory effect on tumor cell invasion in vitro. All these biological effects of SB were reversible and were attenuated following SB withdrawal.

\section{Materials and methods}

Materials and reagents. SB (cat no. 193-01152; reagent grade), trichostatin A (TSA; cat. no. 203-17561), methotrexate (cat. no. 139-13571) and N-acetyl-L-cysteine (NAC; cat. no. 015-05132; ROS scavenger) were purchased from Wako Pure Chemical Industries, Ltd. (Osaka, Japan). Anti-focal adhesion kinase (FAK) polyclonal antibody 
(cat no. sc-1688) and antibodies against p16 (cat no. sc166760), p21 (cat no. sc-166630), p27 (cat no. sc-1641), p53 (cat no. sc-47698), SIRT1 (cat no. sc-74504) MLC20 (cat no. sc-9449) and $\beta$-actin (cat no. sc-47778) were purchased from Santa Cruz Biotechnology, Inc. (Dallas, TX, USA). Anti-phospho (p)-tyrosine pY397-FAK (cat no. 44-624G) and pY577-FAK (cat no. 44-614G) polyclonal antibodies were purchased from Thermo Fisher Scientific, Inc. (Waltham, MA, USA). Anti-p-myosin light chain 20 (p-MLC20) antibody was provided by Dr Fumio Matsumura (Rutgers University, New Brunswick, NJ, USA). All other chemicals used were commercial products from Wako Pure Chemical Industries, Ltd. SB (200 mM stock) and TSA (1 mM stock) were dissolved in phosphate-buffered saline (PBS) and diluted in the cell media prior to use.

Cell culture. The human GB cell line A172 was obtained from the American Type Culture Collection (Manassas, VA, USA). Cells were maintained in Dulbecco's modified Eagle's medium (DMEM) supplemented with $10 \%$ fetal bovine serum (FBS; Thermo Fisher Scientific, Inc.) at $37^{\circ} \mathrm{C}$ in a humidified incubator with $5 \% \mathrm{CO}_{2}$.

Cell proliferation assay and cell cycle analysis. Cell proliferation was analyzed following a previously described protocol (13). Briefly, A172 cells were seeded at a density of $5 \times 10^{3}$ cells/well onto 6-well plates and cultured in DMEM with $10 \% \mathrm{FBS}$ at $37^{\circ} \mathrm{C}$ for $24 \mathrm{~h}$. Cells were then treated with SB $(0,0.5,1,2$ and $4 \mathrm{mM})$ for 7 days. Following 4 days treatment, a subset of A172 cells treated with $2 \mathrm{mM} \mathrm{SB}$ were washed with PBS and cultured for a further 4 days in DMEM with $10 \%$ FBS without SB. For all cells, the culture medium was replenished every $2 \mathrm{~d}$ and cell numbers were recorded every $24 \mathrm{~h}$ using a WST-8 assay (cat no. 341-07761; Wako Pure Chemical Industries, Ltd.). Cell cycle analysis was performed using a BD FACSCalibur flow cytometer (BD Biosciences, Franklin Lakes, NJ, USA), according to the manufacturer's protocol. Briefly, A172 cells were cultured with indicated concentrations of SB, 10, $100 \mathrm{nM}$ TSA or $0.1,1 \mathrm{mM}$ methotrexate for $4 \mathrm{~d}$, then collected and resuspended in $300 \mu \mathrm{l}$ of PBS. After, $700 \mu \mathrm{l}$ of $100 \%$ ethanol was added and kept on ice for 30 min to fix the cells. The fixed cells were resuspended in PBS supplemented with $100 \mu \mathrm{g} / \mathrm{ml}$ of RNase A and $50 \mu \mathrm{g} / \mathrm{ml}$ of propidium iodide (PI) at room temperature for $1 \mathrm{~h}$. The PI stained cells were analyzed according to standard FACS protocol for cell cycle analysis with BD FACStation (v6.1, BD Biosciences, Franklin Lakes, NJ, USA).

Western blot analysis. Cell lysates were prepared with Laemmli's sample buffer ( $1 \mu 1 / 10^{4}$ cells) and $10 \mu \mathrm{g} /$ lane [determined using bicinchoninic acid protein assay kit (cat no. T9300A; Takara Biotechnology Co., Ltd., Dalian, China) according to the manufacturers protocol] was loaded onto 7.5-12.5\% SDS polyacrylamide gel, and western blot analysis was performed as previously described (13) on A172 cells treated with $2 \mathrm{mM} \mathrm{SB}$, prior to and following SB washout. Proteins were electrically transferred to nitrocellulose membranes, then the membranes were incubated with the following primary antibodies: FAK, p16, p21, p27, p53, SIRT1, MLC20 and $\beta$-actin (all at a dilution of 1:1,000), and
pY397FAK, pY-577FAK and p-MLC20 (all 1:100) at $4^{\circ} \mathrm{C}$ overnight. The membranes were then incubated with were alkaline phosphatase (AP)-conjugated anti-rabbit IgG (dilution, 1:7,500; cat. no. S3731; Promega Corporation, Madison, WI, USA) or AP-conjugated anti-mouse IgG (dilution, 1:7,500; cat. no. S3721; Promega Corporation) secondary antibodies for $30 \mathrm{~min}$ at room temperature. Final detection was performed using Western Blue stabilized substrate for alkaline phosphatase (cat. no. S3841, Promega Corporation). Band intensity was analyzed using Image $1.38 \mathrm{e}$ software (National Institutes of Health, Bethesda, MD, USA).

RNA isolation and reverse transcription polymerase chain reaction $(R T-P C R)$. Total RNA was extracted and purified using TRIzol reagent (Invitrogen; Thermo Fisher Scientific, Inc.), according to the manufacturer's protocol. A total of $1 \mu \mathrm{g}$ RNA was subsequently used as a template for reverse transcription using a High-Capacity cDNA Reverse Transcription kit (Applied Biosystems; Thermo Fisher Scientific, Inc.) following the manufacturer's protocol. PCR was performed using Taq DNA Polymerase (Promega Corporation) with the following gene-specific primers: p21, forward, 5'-TTATGA AАT TCACCСССТTT-3'; and reverse, 5'-GCCCCTTCA AAGTGCCATCT-3'; p27, forward, 5'-AGATGTCAAACG TGCGAGTG-3'; and reverse, 5'-TCAGTCTTTGGGTCC ACCAA-3'; p53, forward, 5'-GGGCCCACTTCACCGTAC TA-3'; and reverse, 5'-TCTACCTAACCAGCTGCCCA-3'; and GAPDH, forward, 5'-AAAGGGAAGCTGACAGGGAT-3' and reverse, 5'-GTGGGTATCACCGAGGAAGT-3'. GAPDH functioned as the reference gene. Thermocycling conditions were $95^{\circ} \mathrm{C}$ for $30 \mathrm{sec}$ for denaturation, $60-65^{\circ} \mathrm{C}$ for $30 \mathrm{sec}$ for annealing and $68^{\circ} \mathrm{C}$ for $1 \mathrm{~min}$ for extension and cycle numbers were $30-35$ cycles.

Immunofluorescence microscopy. Immunofluorescence analysis was performed following a previously described protocol (13). Briefly, A172 cells were cultured with 0-4 mM $\mathrm{SB}$ on type I collagen-coated 2-well chamber slides (BD Biosciences). After $48 \mathrm{~h}$, the cells were fixed with $1 \%$ paraformaldehyde at room temperature for $1 \mathrm{~h}$ in PBS and then permeabilized with $0.2 \%$ TritonX-100 in PBS. Non-specific blocking was performed in $0.1 \%$ bovine serum albumin (Wako Pure Chemical Industries, Ltd.) at room temperature for $1 \mathrm{~h}$. Cells were incubated with primary antibody, Anti-p-tyrosine pY397-FAK (dilution, 1:100) at $4^{\circ} \mathrm{C}$ overnight, followed by incubation with Alexa 488 anti-rabbit immunoglobulin G (dilution, 1:1,000; cat no. A-11008; Molecular Probes; Thermo Fisher Scientific, Inc.) secondary antibody, at room temperature for $1 \mathrm{~h}$ in dark room. Images were obtained using the Olympus BX50 fluorescence microscope (magnification, $\mathrm{x} 40$; numerical aperture, 0.6; Olympus Corporation, Tokyo, Japan). Images were analyzed and processed for presentation by adjusting brightness and contrast using ImageJ 1.38 software (National Institutes of Health, Bethesda, MD, USA).

Effects of SB on cell motility and invasion. A172 cells were pretreated with $0,0.25,0.5,1,2$ and $4 \mathrm{mM}$ of SB for $48 \mathrm{~h}$. The cell motility assay was performed for $16 \mathrm{~h}$ using 24-well Bio-Coat cell migration chambers (BD Biosciences) using 
$0.5 \%$ FBS as a chemoattractant, following a previously described protocol (13). The cell invasion assay was performed using Matrigel-coated polyethylene terephthalate membranes for $16 \mathrm{~h}$. Cells at a density of $2 \times 10^{5}$ cells/well were inserted into the upper chamber. The migrating cells on the lower side of the filter were fixed with $70 \%$ ethanol at room temperature for $1 \mathrm{~h}$, prior to being stained with Giemsa solution (Wako Pure Chemical Industries, Ltd.) at room temperature for $10 \mathrm{~min}$ and counted using Olympus IX70 phase contrast microscopy with a x10 objective lens. For cell area analysis, cell images were captured using Olympus IX70 phase contrast microscopy with a $\mathrm{x} 10$ objective lens. Cell periphery was circled then the inner area was calculated with ImageJ 1.38 e software. A minimum of 100 cells/each experiment were measured.

$\beta$-gal staining assay. The $\beta$-gal staining assay was performed using a Senescence $\beta$-Galactosidase Staining kit (cat. no. 9860; Cell Signaling Technology, Inc., Danvers, MA, USA). Briefly, A172 cells were cultured with $0,0.25,0.5,1$, 2 or $4 \mathrm{mM} \mathrm{SB}$, or 25,50 or $100 \mathrm{nM}$ TSA at $37^{\circ} \mathrm{C}$ for 4 days in $35 \mathrm{~mm}$ plastic culture plates, and fixed with $10 \%$ formaldehyde at room temperature for $1 \mathrm{~h}$. Plates were rinsed twice with PBS, subsequently the $\beta$-gal staining solution containing $\mathrm{X}$-gal as a substrate was added and plates were incubated at $37^{\circ} \mathrm{C}$ overnight in a dry incubator. $\beta$-gal-positive cells were then counted using Olympus IX70 phase contrast microscopy with $\mathrm{x} 10$ objective lens and the percentage of positive cells was calculated.

Proteasome activity assay. The proteasome activity assay was performed using a Proteasome Activity assay kit (cat. no. ab107921; Abcam, Tokyo, Japan) following the manufacturer's protocol. A172 cells were treated with $2 \mathrm{mM} \mathrm{SB}$ for $48 \mathrm{~h}$. The chymotrypsin-like function of A172 cell lysate for a 7-Amino-4-methyl-coumarin-tagged peptide substrate (proteasome substrate Succ-LLVY-AMC) was compared with that of the positive control Jurkat cell lysate in the presence of the proteasome inhibitor MG132 $(2 \mu \mathrm{M})$.

Transient RNA interference. Small interfering (si)RNA against human p21 (CDKN1A, SignalSilence p21 Waf1/Cip1 siRNA) was obtained from Cell Signaling Technology, Inc. A siRNA-control was designed for Photinus Pyralis GL3 luciferase as previously described and obtained from Hokkaido System Science Co., Ltd. (Sapporo, Japan) (14). This gene is not present in mammalian cell transcripts and therefore the luciferase siRNA-control minimizes the off-targeting effect (14). A172 cells were transfected with siRNA-p21 or siRNA-control at a final concentration of $50 \mathrm{nmol} / 1$ using Lipofectamine 2000 (Invitrogen; Thermo Fisher Scientific, Inc.). Cell lysates were prepared for western blotting $72 \mathrm{~h}$ after transfection, performed as aforementioned. Transfected cells underwent staining for $\beta$-gal. Cell motility and invasion assays, as well as cell cycle analyses were also performed.

Statistical analysis. Data are expressed as the mean \pm standard deviation. Differences were determined by analysis of variance with Bonferroni's test as a post-hoc test in JMP software (version 11; SAS Institute, Inc., Cary, NC, USA).
$\mathrm{P}<0.01$ was considered to indicate a statistically significant difference.

\section{Results}

Effect of SB on the proliferation of A172 human glioblastoma cells. Physiological concentrations of SB (0.5-4 mM) inhibited the proliferation of the human GB A172 cells in a dose-dependent manner (Fig. 1A). In addition, cell cycle analysis identified an increase in the percentage of cells in the G0/G1-phase following SB treatment, suggesting G1/S arrest. This inhibitory effect of SB on A172 cell proliferation and cell cycle progression was reversible and attenuated following wash-out of the drug [Fig. 1B (orange line) and Fig. 1C]. No sub-G1 apoptotic population was observed following FACS analysis of the A172 cells treated with SB. By contrast, FACS analysis identified apoptotic A172 cells following the treatment of cells with the HDAC inhibitor TSA or the chemotherapy drug methotrexate (data not shown).

Effect of SB and the HDAC inhibitor TSA on SA $\beta$-gal staining. SB also induced positive staining for SA $\beta$-gal in A172 cells (Fig. 2A). This positive staining for $\beta$-gal, indicating cellular senescence, was SB dose-dependent (Fig. 2B). However, the HDAC inhibitor TSA did not induce any positive staining for $\beta$-gal (Fig. 2B). To elucidate the mechanism associated with this cell cycle arrest, the expression of cell cycle regulator proteins was assessed.

SB increased the p21 protein level. A172 cells treated with 2 mM SB exhibited elevated levels of p21,p27 and p53 and this increase in expression was time-dependent (Fig. 3A); however, levels of p21 mRNA were decreased $24 \mathrm{~h}$ after treatment with SB (Fig. 3B). Since A172 cells harbor wild-type p53 (15), it was deduced that the p53-p21 axis functioned in the cells. The results of the present study suggest that $24 \mathrm{~h}$ treatment with SB stabilizes the expression of the three cell cycle regulator proteins p21, p27 and p53 in A172 cells. Although levels of p21 mRNA decreased, the levels of p27 and p53 mRNA were unaltered. Therefore, it is likely that the post-translational protein stabilization of p21, p27 and p53 induced by SB treatment is the primary mechanism responsible for the results of the present study. The present study therefore assessed the potential inhibitory activity of SB against the proteasome compared with that of MG132, a specific proteasome inhibitor. SB did not exhibit any direct inhibitory effect on the proteasome in this proteasome activity assay (data not shown).

Effect of SB on the motility, invasion and morphology of A172 cells. SB also inhibited cellular motility and invasion in a dose-dependent manner and affected the morphology of A172 cells (Fig. 4). Increased cell area and adherence to the extracellular matrix was observed following treatment with SB (Fig. 4C). Since 48 h SB treatment did not affect cellular proliferation or apoptosis (Fig. 1), it could be concluded that the decreased number of infiltrated cells was due to a decrease in cell motility following SB treatment and not due to a decrease in total cell number. In addition, the phosphorylation of FAK (Y397 site) was increased, while that of MLC20 (S19 site) remained unaltered (Fig. 4B). 


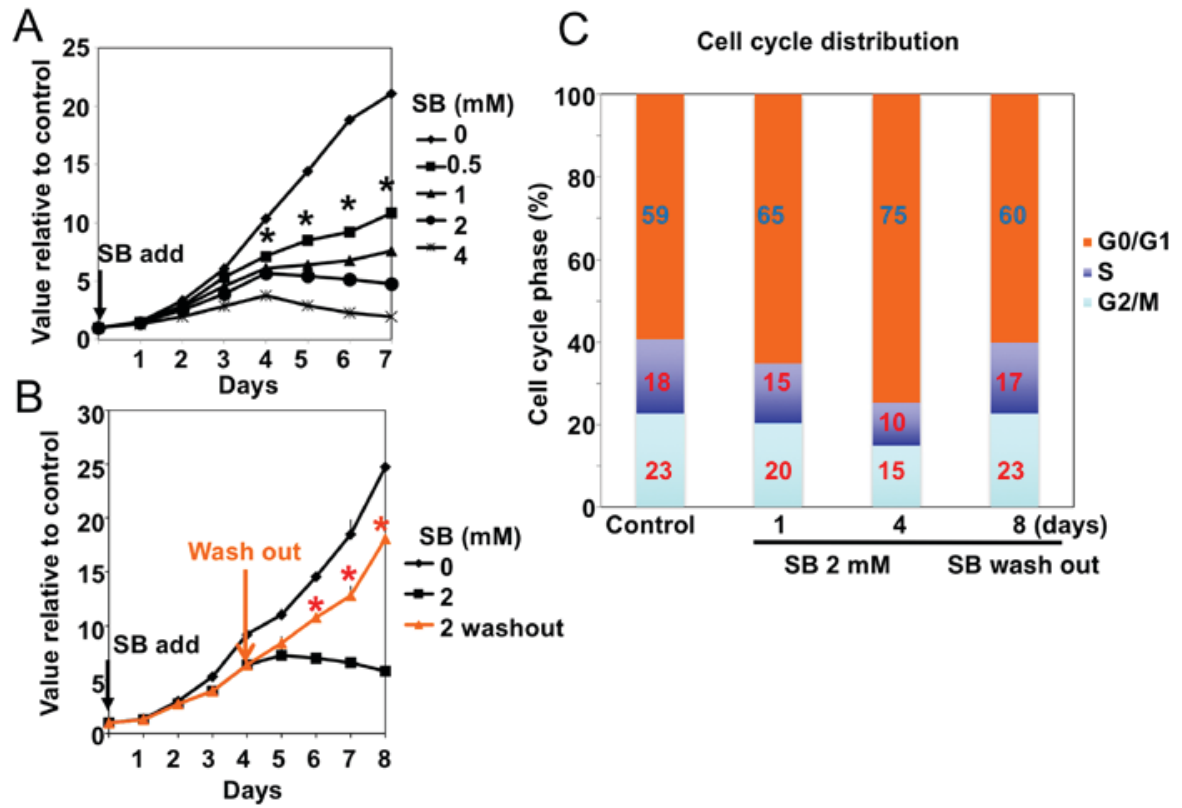

Figure 1. Effect of SB on the proliferation of A172 human glioblastoma cells. (A) In vitro proliferation assay of A172 cells following treatment with different concentrations of SB $(0.0,0.5,1.0,2.0$ and $4.0 \mathrm{mM})$. Results are presented as the mean \pm standard deviation $(\mathrm{n}=3)$. " $\mathrm{P}<0.01 \mathrm{vs}$. treatment with 0 mM SB . (B) Reversibility of SB inhibitory effect. A total of 4 days following treatment with $2 \mathrm{mM} \mathrm{SB}$, A172 cells were washed with media without SB and cultured for 4 additional days. The results are presented as the mean \pm standard deviation ( $\mathrm{n}=3$ ). ${ }^{*} \mathrm{P}<0.01$ vs. treatment with $2 \mathrm{mM}$ SB. (C) Cell cycle analysis of the A172 cells in (B) using a BD FACSCalibur system. SB, sodium butyrate.
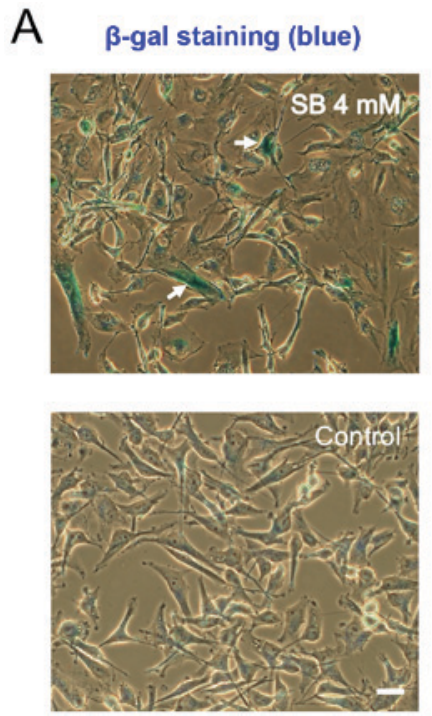

B

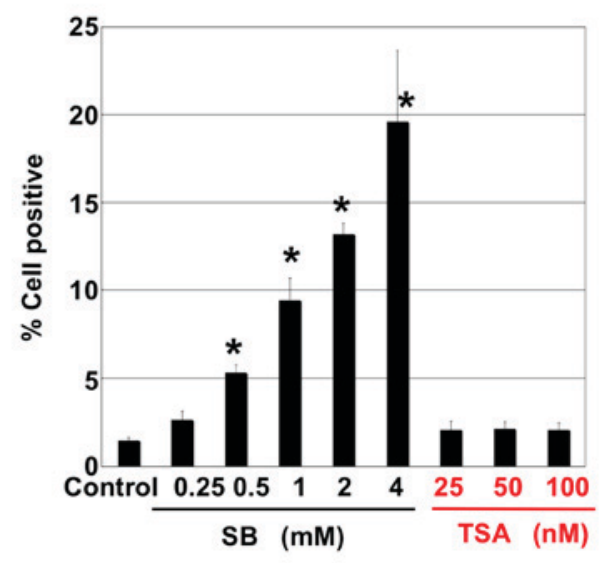

Figure 2. Effect of SB and the HDAC inhibitor TSA on SA $\beta$-gal staining. (A) A172 cells treated with SB (0-4 mM) or TSA (25-100 nM) for 4 days were stained with SA $\beta$-gal. $\beta$-gal-positive cells are indicated by white arrows (scale bar=20 $\mu \mathrm{m}$ ). (B) $\beta$-gal-positive cells in (A) were analyzed and counted. Results are presented as the mean \pm standard deviation $(\mathrm{n}=4)$; ${ }^{\prime} \mathrm{P}<0.01$ vs. control. SA $\beta$-gal, senescence-associated $\beta$-galactosidase; SB, sodium butyrate; HDAC, histone deacetylase; TSA, trichostatin A.

The results of the immunofluorescence analysis indicated that SB dose-dependently induced an increase in the amount of focal adhesions at the cell peripheries (white arrows, stained with anti-pY397-FAK antibodies; Fig. 5) and increased cell spreading area on the substratum. The effects of SB on the expression and phosphorylation of FAK were reversible and were attenuated following the washing out of SB from the cell culture (Fig. 6A). Cellular motility also recovered 3-4 days following SB wash out (Fig. 6B). p21 involved SB induced A172 cellular senescence but not involved cellular motility. The present study focused on the underlying mechanism of SB regarding its effects on cellular senescence and motility. Knockdown of p21 expression was achieved by transfection with siRNA specific for the human p21 gene CDKN1A. The inhibition of p21 expression was confirmed by western blotting and assessed following the treatment of cells with $2 \mathrm{mM} \mathrm{SB}$ (Fig. 7A). Knockdown of p21 expression did not affect the expression levels of p53, p27 and p16 (Fig. 7A). In contrast, knockdown 
A

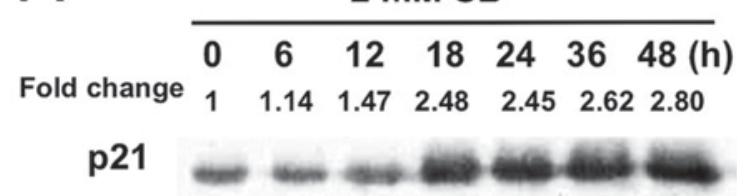

$\begin{array}{llllllll}\text { Fold change } & 1 & 1.10 & 2.74 & 2.88 & 3.25 & 2.92 & 3.35\end{array}$

p27

$\begin{array}{llllllll}\text { Fold change } & 1 & 2.40 & 2.09 & 2.18 & 3.55 & 3.42 & 2.95\end{array}$ p53

$\beta$-actin
B

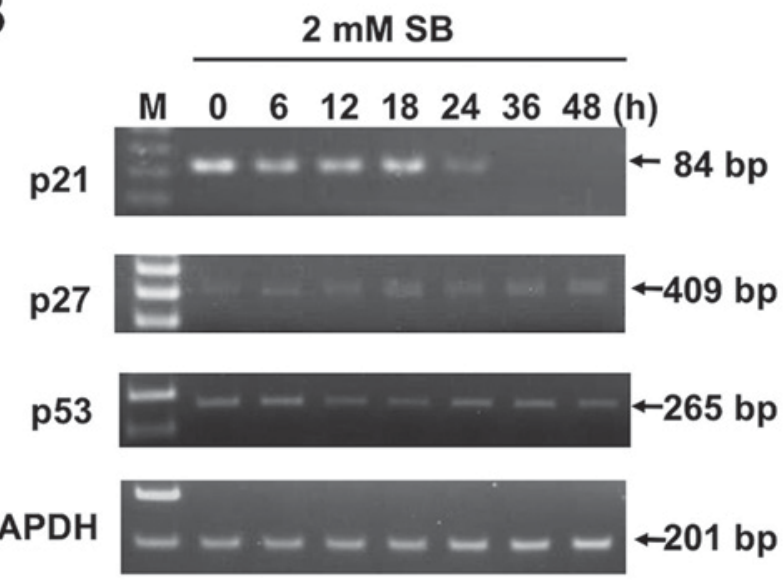

Figure 3. Western blot and RT-PCR analyses of p21 (FK506 binding protein like), p27 (cyclin dependent kinase inhibitor 1B) and p53 mRNA and protein expression in 2-mM SB-treated A172 cells. (A) A172 cells were treated with $2 \mathrm{mM}$ SB for the indicated time and the protein levels of the cell cycle regulators p21, p27 and p53 were analyzed by western blot analysis. (B) A172 cells were treated with 2 mM SB for the indicated time and mRNA levels of p21, p27 and p53 were analyzed by RT-PCR. RT-PCR, reverse transcription-polymerase chain reaction; SB, sodium butyrate; bp, base pairs.
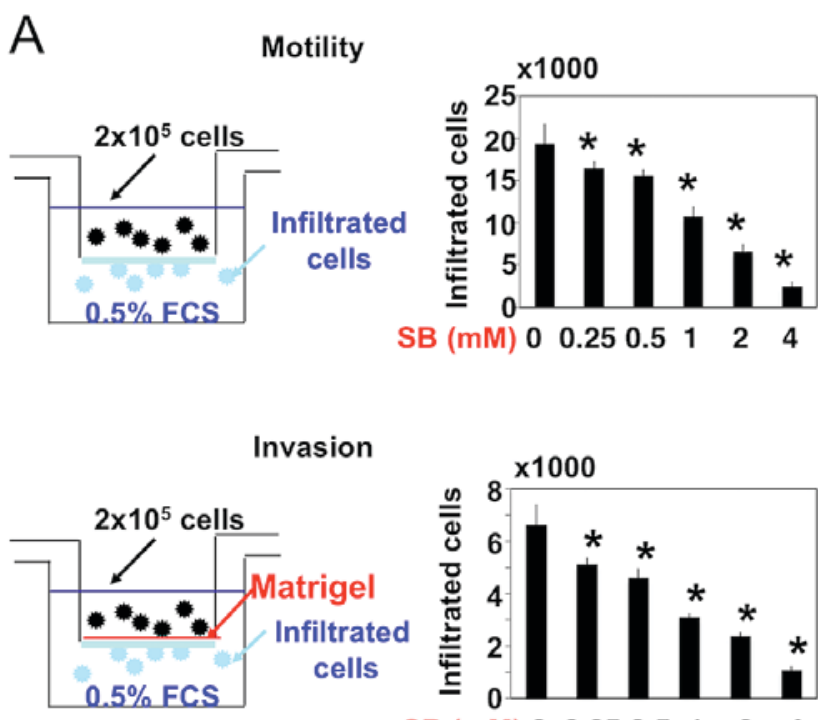

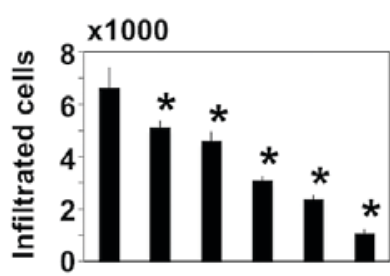

$\mathrm{SB}(\mathrm{mM}) \mathbf{0} \quad 0.250 .5 \quad 1 \quad 2 \quad 4$
B
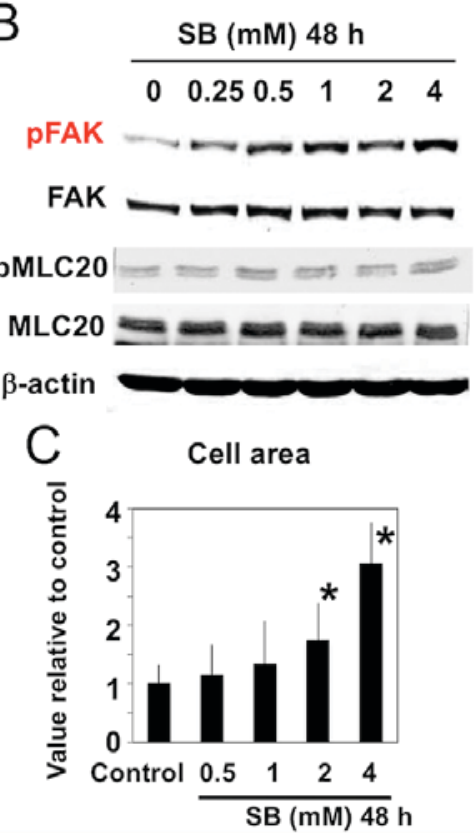

Figure 4. Effect of SB on the motility, invasion and morphology of A172 cells. (A) Upper panel: A172 cells were treated with the indicated concentrations $(0-4 \mathrm{mM})$ of SB for $48 \mathrm{~h}$ and then cell motility was analyzed using with $0.5 \%$ FBS as a chemoattractant. Results are presented as the mean \pm standard deviation ( $\mathrm{n}=6$ ). Lower panel: Cell invasion was analyzed with $0.5 \%$ FBS as a chemoattractant using Matrigel-coated chambers. Results are presented as the mean \pm standard deviation $(n=3)$. ${ }^{*} \mathrm{P}<0.01$ vs. control. (B) A172 cells were treated with the indicated concentrations $(0-4 \mathrm{mM})$ of SB for $48 \mathrm{~h}$ and then the phosphorylation of FAK and MLC20 were analyzed by western blotting. (C) A172 cells were treated with the indicated concentrations ( $0-4 \mathrm{mM})$ of SB for $48 \mathrm{~h}$ and then cell spreading was analyzed by measuring the cell area. Results are presented as the mean \pm standard deviation $(\mathrm{n}=100)$. ${ }^{*} \mathrm{P}<0.01 \mathrm{vs}$. control. $\mathrm{SB}$, sodium butyrate; FBS, fetal bovine serum; FAK, focal adhesion kinase; MLC, myosin light chain; p-, phosphorylated.

of p21 expression partially reversed the inhibition of proliferation and positive SA $\beta$-gal staining, but cell motility remained inhibited (Fig. 7B). Knockdown of p21 expression increased $\mathrm{S}$ phase in the control and SB treated cells using cell cycle analysis (Fig. 7C). However, the increase in FAK phosphorylation induced by $2 \mathrm{mM}$ SB was unaffected following knockdown of p21 (Fig. 7A). These results suggest that upregulation of p21 is implicated in the cellular senescence, but not the inhibition of cell motility, that is induced by SB.

\section{Discussion}

The results of the present study indicated that SB reversibly inhibited A172 cell proliferation and induced cellular senescence by upregulating and stabilizing the cell cycle regulator proteins p21, p27 and p53. SB also reversibly inhibited cellular motility and invasion. Downregulating p21 using specific siRNA partially reversed the proliferation-inhibiting effect of SB on cell cycle analysis and $\beta$-gal staining. By contrast, cell motility, cell spreading and the phosphorylation 

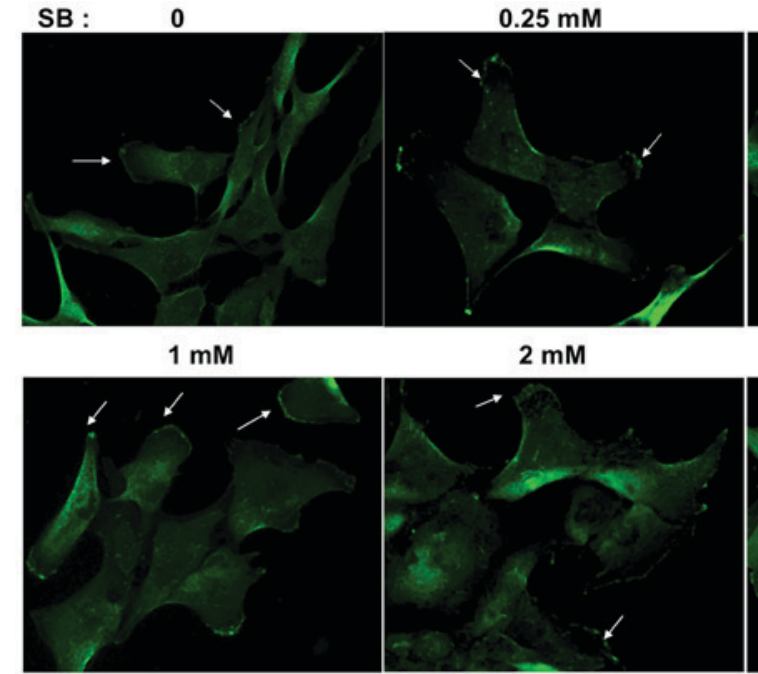

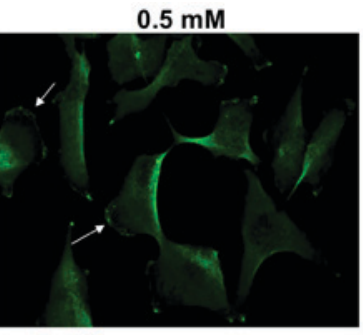

$4 \mathrm{mM}$

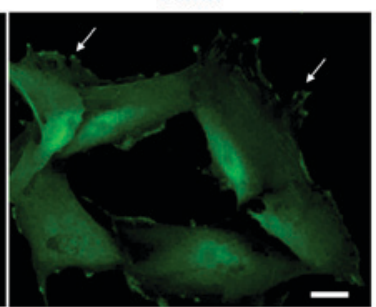

Green : FAK pY397

Figure 5. Effect of SB on A172 cell morphology and intracellular FAK and MLC20 phosphorylation. Immunostaining of A172 cells treated with the indicated concentrations (0-4 mM) of SB for $48 \mathrm{~h}$ using phospho-specific pY397 FAK antibody. White arrows indicate the focal adhesions at the cell peripheral regions. Scale bar $=20 \mu \mathrm{m}$. SB, sodium butyrate; FAK, focal adhesion kinase; MLC, myosin light chain.
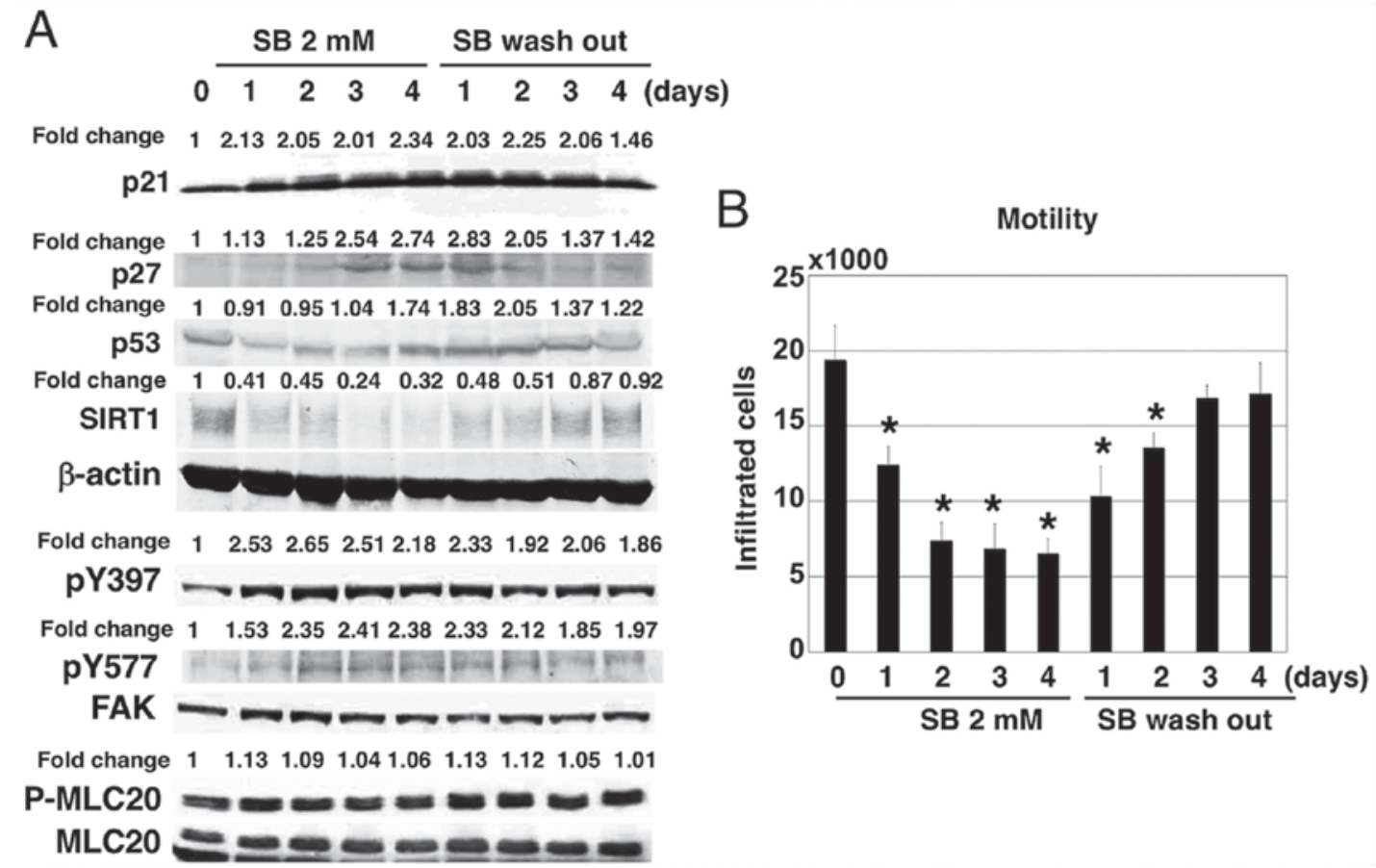

Figure 6. Reversibility of the effects of SB in A172 cells. (A) At 4 days after treatment with $2 \mathrm{mM} \mathrm{SB}$, A172 cells were washed in media without SB and cultured for 4 additional days. The effects on the expression of p21 (FK506 binding protein like), p27 (cyclin dependent kinase inhibitor 1B), p53, SIRT1, pY397/pY577 FAK and p-MLC20 were determined by western blotting. (B) Cell motility was measured in A172 cells treated as in (A). Results are presented as the mean \pm standard deviation $(\mathrm{n}=6)$. ${ }^{*} \mathrm{P}<0.01$ vs. control. $\mathrm{SB}$, sodium butyrate; SIRT, sirtuin; FAK, focal adhesion kinase; $\mathrm{p}-$, phosphorylated; MLC, myosin light chain.

of FAK remained decreased following p21 knockdown in A172 cells.

SB exhibits HDAC-inhibiting activity. However, TSA (a typical HDAC inhibitor) did not promote cellular senescence or reversibly inhibit cellular proliferation in A172 cells, and did not induce cell apoptosis. Previous studies by our group used similar assays to evaluated the effect of SB in various other tumor cell lines, including human fibrosarcoma (HT-1080), human breast cancer (MDA-MB-231; unpublished) and rat mammary cancer (Walker 256; manuscript in preparation). In all cases, SB reversibly inhibited cell proliferation, increased cell adhesion, and inhibited cell motility and invasion. Furthermore, the effect of SB on normal primary cultured cells was assessed and it was identified that SB did not affect cell proliferation much. Therefore, SB may potentially be used as an anti-cancer therapy, although continuous treatment may be required. Using an osmotic pump to maintain the drug concentration, 


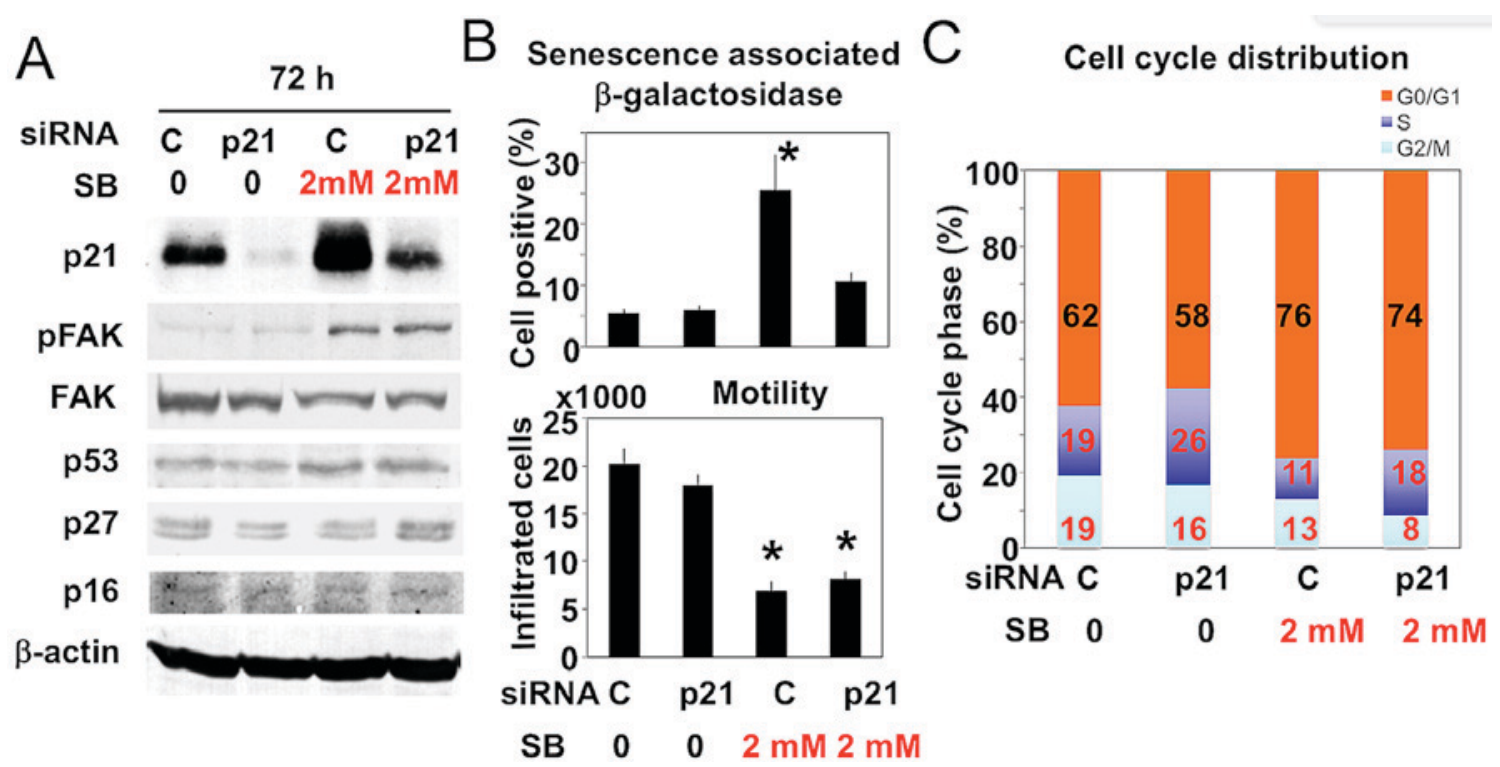

Figure 7. Effect of SB on the levels of p21 (FK506 binding protein like), p53, p27 (cyclin dependent kinase inhibitor 1B), p16 and pFAK in p21 knockdown and control A172 cells. (A) Knockdown of p21 was achieved by transfection with specific siRNA against human p21 for $72 \mathrm{~h}$. The effect of $2 \mathrm{mM}$ SB on the levels of p21 (FK506 binding protein like), p53, p27 (cyclin dependent kinase inhibitor 1B), p16 and FAK phosphorylation in control and p21-knockdown cells was determined using western blot analysis. (B) $\beta$-galactosidase positive staining ( $\mathrm{n}=4$; upper panel); and cell motility (lower panel; $n=6)$ were measured in the A172 cells in (A). Results are presented as the mean \pm standard deviation. ${ }^{*} \mathrm{P}<0.01$ vs. control. (C) Cell cycle analysis of the A172 cells in (A) using a BD FACScalibur system. SB, sodium butyrate; p-, phosphorylated; FAK, focal adhesion kinase; si, small interfering; C, control.

as reported previously (13), the intrathecal administration of SB into rat cerebrospinal space was performed in a previous study by our group, which inhibited the invasion of Walker 256 cells (a syngeneic rat mammary cancer cell line) into the brain parenchyma and increased the survival rate of rats (manuscript in preparation).

The mechanism corresponding to the inhibition of motility induced by SB is currently under investigation. It has been reported that cancer motility and invasion is primarily regulated by the Rho family of small GTP-binding proteins (including RhoA and cell division cycle 42) and Rho-associated kinase (ROCK), and that the downstream molecular motor activity of myosin, determined by the phosphorylation of MLC20, is crucial (16). The specific ROCK inhibitor Y-27632 inhibits the motility, invasion and adhesion of hepatoma $(16,17)$ and breast cancer cells $(13,18)$. Our group has also demonstrated that the invasive phenotype of murine osteosarcoma cells is positively associated with increased FAK levels (19). In the present study, the effects of SB were unusual, particularly regarding the increases in FAK phosphorylation, cell spreading and cell area associated with decreased cell motility. Therefore, novel molecular targets of SB within tumor cells should be evaluated. SB induced multiple metabolic changes in A172 cells; therefore, metabolic pathways associated with isocitrate dehydrogenase 1 and branched chain aminotransferase, which have been implicated as critical targets in oncogenesis and progression of malignant glioma (20), may be associated with the effect of SB on glioma cell motility. Further studies by our group aim to analyze the metabolome alterations induced by SB.

Previous studies have focused on the effect of butyrate on microRNAs (miRs). Pant et al (21) reported that miR-22 and SB inhibited sirtuin 1 (SIRT-1) expression, enhanced reactive oxygen species (ROS) release in hepatic cancer cells and induced cellular apoptosis. Similarly, the present study demonstrated that treatment with SB reversibly inhibited the expression of SIRT-1 in A172 cells; however, an ROS scavenger (N-acetyl-L-cysteine (NAC)) did not affect SB-induced A172 cell senescence (data not shown). Han et al (22) reported that SB induced miR-203 expression and that the miR-203 target gene neural precursor cell expressed developmentally downregulated 9 was associated with the SB-induced inhibition of colony formation and invasion in colorectal cancer cells. Further studies into the targeting activity of SB besides its HDAC-inhibiting function are required.

It was previously reported that irradiation induced GB cells to senescence (23) and that anti-vascular endothelial growth factor A-targeting therapy induced senescence-associated cell death (24). p21 protein (FK506 binding protein like)-dependent cytotoxicity was suggested in these cases $(23,24)$. Therefore, the present study hypothesized that SB-induces senescence via a similar pathway.

In conclusion, SB inhibited A172 cell proliferation, induced cellular senescence and inhibited cell invasion, potentially via a mechanism associated with upregulation of $\mathrm{p} 21$. It may therefore be developed as a novel treatment for GB.

\section{Acknowledgements}

The authors acknowledged that the preliminary work of the present study was reported at the American Association for Cancer Research (AACR) annual meeting on April 9, 2013, and appeared in an AACR article as the meeting abstract (25). The authors would like to thank Dr Kiyoko Yoshioka (Osaka Medical Center for Cancer and Cardiovascular Diseases, Osaka, Japan) for her technical assistance. The present study was supported by the Japan Society for the Promotion of Science (grant no. $15 \mathrm{~K} 15560$ to KI). 


\section{References}

1. Stupp R, Mason WP, van den Bent MJ, Weller M, Fisher B, Taphoorn MJ, Belanger $\mathrm{K}$, Brandes AA, Marosi C, Bogdahn U, et al: Radiotherapy plus concomitant and adjuvant temozolomide for glioblastoma. N Engl J Med 352: 987-996, 2005

2. Stupp R, Hegi ME, Mason WP, van den Bent MJ, Taphoorn MJ, Janzer RC, Ludwin SK, Allgeier A, Fisher B, Belanger K, et al: Effects of radiotherapy with concomitant and adjuvant temozolomide versus radiotherapy alone on survival in glioblastoma in a randomised phase III study: 5-year analysis of the EORTC-NCIC trial. Lancet Oncol 10: 459-466, 2009.

3. Gzell C, Back M, Wheeler H, Bailey D and Foote M: Radiotherapy in Glioblastoma: The Past, the present and the future. Clin Oncol (R Coll Radiol) 29: 15-25, 2017.

4. Nakagawa H, Yamada M, Kanayama T, Tsuruzono K, Miyawaki Y, Tokiyoshi K, Hagiwara Y and Hayakawa T: Myelin basic protein in the cerebrospinal fluid of patients with brain tumors. Neurosurgery 34: 825-833, 1994.

5. Blumenthal DT, Gorlia T, Gilbert MR, Kim MM, Burt Nabors L, Mason WP, Hegi ME, Zhang P, Golfinopoulos V, Perry JR, et al: Is more better? The impact of extended adjuvant temozolomide in newly diagnosed glioblastoma: A secondary analysis of EORTC and NRG Oncology/RTOG. Neuro Oncol 19: 1119-1126, 2017.

6. Heerdt BG, Houston MA and Angenlicht LH: Potentiation by specific short-chain fatty acids of differentiation and apoptosis in human colonic carcinoma cell lines. Cancer Res 54: 3288-3293, 1994.

7. Hague A, Elder DJ, Hicks DJ and Paraskeva C: Apoptosis in colorectal tumour cells: Induction by the short chain fatty acids butyrate, propionate and acetate and by the bile salt deoxycholate. Int J Cancer 60: 400-406, 1995.

8. Farrow B, Rychahou P, O'Connor KL and Evers BM: Butyrate inhibits pancreatic cancer invasion. J Gastrointest Surg 7: 864-870, 2003.

9. Wang XM, Li J and Evers BM: Inhibition of proliferation, invasion and adhesion of liver cancer cells by 5 -azacytidine and butyrate. Anticancer Res 19: 2901-2906, 1999.

10. Engelhard HH, Duncan HA, Kim S, Criswell PS and Van Eldik L: Therapeutic effect of sodium butyrate on glioma cells in vitro and in the rat C6 glioma model. Neurosurgery 48: 616-625, 2001.

11. Hague A, Manning AM, Hanlon KA, Huschtscha LI, Hart D and Paraskeva C: Sodium butyrate induces apoptosis in human colonic tumour cell lines in a p53-independent pathway: Implications for the possible role of dietary fibre in the prevention of large-bowel cancer. Int J Cancer 55: 498-505, 1993.

12. Medina V, Edmonds B, Young GP, James R, Appleton S and Zalewski PD: Induction of caspase-3 protease activity and apoptosis by butyrate and trichostatin A (inhibitors of histone deacetylase): Dependence on protein synthesis and synergy with a mitochondrial/cytochrome c-dependent pathway. Cancer Res 57: 3697-3707, 1997.

13. Nakagawa H, Yoshioka K, Miyahara E, Fukushima Y, Tamura M and Itoh K: Intrathecal administration of Y-27632, a specific Rho-associated kinase inhibitor, for rat neoplastic meningitis. Mol Cancer Res 3: 425-433, 2005.
14. Naka N, Takenaka S, Araki N, Miwa T,Hashimoto N, Yoshioka K, Joyama S, Hamada K, Tsukamoto Y, Tomita Y, et al: Synovial sarcoma is a stem cell malignancy. Stem Cells 28: 1119-1131, 2010.

15. Park CM, Park MJ, Kwak HJ, Moon SI, Yoo DH, Lee HC, Park IC, Rhee CH and Hong SI: Induction of p53-mediated apoptosis and recovery of chemosensitivity through p 53 transduction in human glioblastoma cells by cisplatin. Int J Oncol 28: 119-125, 2006.

16. Itoh K, Yoshioka K, Akedo H, Uehata M, Ishizaki T and Narumiya S: An essential part for Rho-associated kinase in the transcellular invasion of tumor cells. Nat Med 5: 221-225, 1999.

17. Yoshioka K, Nakamori S and Itoh K: Overexpression of small GTP-binding protein RhoA promotes invasion of tumor cells. Cancer Res 59: 2004-2010, 1999.

18. Yoshioka K, Foletta V, Bernard O and Itoh K: A role for LIM-kinase in cancer invasion. Proc Natl Acad Sci USA 100: 7247-7252, 2003.

19. Yui Y, Itoh K, Yoshioka K, Naka N, Watanabe M, Hiraumi Y, Matsubara H, Watanabe K, Sano K, Nakahata T and Adachi S: Mesenchymal mode of migration participates in pulmonary metastasis of mouse osteosarcoma LM8. Clin Exp Metastasis 27: 619-630, 2010

20. Karsy M, Guan J, Cohen AL, Jensen RL and Colman H: New molecular considerations for Glioma: IDH, ATRX, BRAF, TERT, H3 K27M. Curr Neurol Neurosci Rep 17: 19, 2017.

21. Pant K, Yadav AK, Gupta P, Islam R, Saraya A and Venugopal SK: Butyrate induces ROS-mediated apoptosis by modulating miR-22/SIRT-1 pathway in hepatic cancer cells. Redox Biol 12: 340-349, 2017.

22. Han R, Sun Q, Wu L, Zeng P and Zhao G: Sodium butyrate upregulates miR-203 expression to exert Anti-proliferation effect on colorectal cancer cells. Cell Physiol Biochem 39: 1916-1929, 2016.

23. Jeon HY, Kim JK, Ham SW, Oh SY, Kim J, Park JB, Lee JY, Kim SC and Kim H: Irradiation induces glioblastoma cell senescence and senescence-associated secretory phenotype. Tumor Biol 37: 5857-5867, 2016.

24. Morelli MB, Amantini C, Nabissi M, Cardinali C, Santoni M, Bermadini G, Santoni A and Santoni G: Axitinib induces senescence-associated cell death and necrosis in glioma cell lines: The proteasome inhibitor, bortezomib, potentiates axitinib-induced cytotoxicity in a $\mathrm{p} 21$ (Waf/Cip1) dependent manner. Oncotarget 8: 3380-3395, 2017.

25. Itoh K, Yoshioka $\mathrm{K}$ and Nakagawa $\mathrm{H}$ : Sodium butyrate induced cellular senescence and inhibited invasion of cancer cells with distinct mechanism. Cancer Res 73 (8 Suppl): S2624, 2013.

This work is licensed under a Creative Commons Attribution-NonCommercial-NoDerivatives 4.0 International (CC BY-NC-ND 4.0) License. 